\title{
Editorial
}

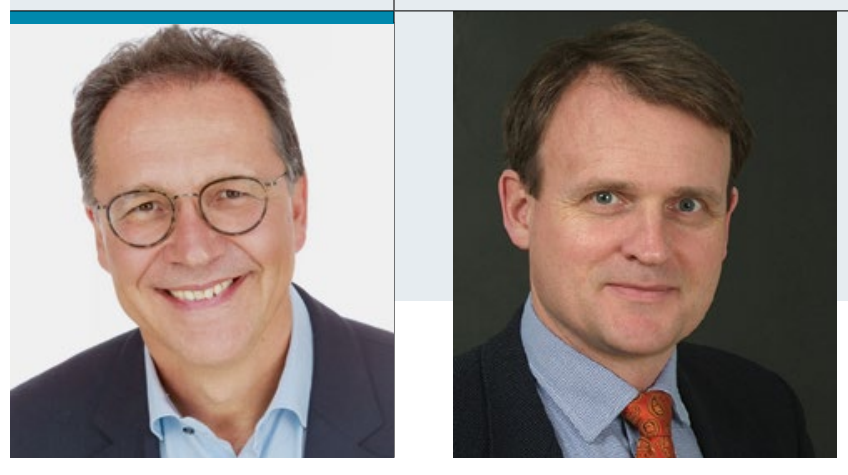

„Seltene Allergene stellen in

Diagnostik und Versorgung

allergiekranker Patienten ein

zunehmendes Problem dar."

Prof. Dr. Ludger Klimek, Zentrum für Rhinologie und Allergologie Wiesbaden

Prof. Dr. Thilo Jakob, Klinik für Dermatologie und Allergologie,

Universitätsklinikum Gießen und Marburg, Standort Gießen

\section{Von Antibiotika, Allergenen und Abstracts}

A ntibiotika gehören zu den meistverordneten Medikamentengruppen und verursachen gleichzeitig überproportional häufig allergische Reaktionen bis hin zur Anaphylaxie. In einer aktuellen, CME-zertifizierten Literaturübersicht in dieser Ausgabe (S. 43) wird die Problematik von Antibiotikaallergien dargestellt. Eine sorgfältige Diagnostik ermöglicht bei positivem Ergebnis sowohl den sicheren Nachweis der Erkrankung als auch bei Ausschluss die Weiterverwendung wichtiger Substanzklassen und kann somit helfen, den unnötigen Einsatz von Reserveantibiotika zu reduzieren.

Prof. Dr. Wolfgang Uter beschreibt - im Rahmen der Allergo-Journal-Serie „Das kleine $1 \times 1$ der Kontaktallergene" - die Inhaltsstoffe von Friseurprodukten, die insbesondere in Haarfärbemitteln, Blondierungen und Wellmitteln enthalten sind (S. 20). Bei Verdacht auf eine Kontaktsensibilisierung sollte mit einer geeigneten Epikutantestreihe wie der „Friseurreihe“ der Deutschen Kontaktallergie Gruppe getestet werden. Des Weiteren wird die aktuelle Empfehlung einer europäischen Haarkosmetikareihe präsentiert.

Prof. Dr. Bettina Wedi zeigt auf, dass nicht steroidale Antirheumatika (NSAR) die zweithäufigsten Auslöser einer Arzneimittelüberempfindlichkeitsreaktion bei Erwachsenen und Kindern darstellen (S. 34). Der Pathomechanismus ist für die meisten Unverträglichkeitsreaktionen auf NSAR nicht im Detail geklärt. Häufig treten Sofortreaktionen auf mit kutaner (Urtikaria/Angioödem) und/ oder respiratorischer Klinik (Rhinitis, Asthma bronchiale) sowie anaphylaktische Reaktionen. Für HNO-Ärzte ist insbesondere die assoziierte Polyposis nasi ein erhebliches klinisches Problem. Diagnostik und Management der NSAR-Überempfindlichkeitsreaktionen werden detailliert aufgezeigt und auf Provokationstestungen mit dem verdächtigen NSAR als Goldstandard verwiesen.
Seltene Allergien stellen in Diagnostik und Versorgung allergiekranker Patienten ein zunehmendes Problem dar. In der Europäischen Union gilt eine Erkrankung als selten, wenn nicht mehr als fünf von 10.000 Menschen davon betroffen sind. Dies gilt für zahlreiche Inhalations- und Nahrungsmittel sowie Kontaktallergene. In der Allergologie sind die Herausforderungen für die Patientenversorgung besonders hoch, da nicht nur die (Immun-) Therapie, sondern auch die (In-vivo-)Diagnostik betroffen sind (S. 94). Der AeDA startet in diesem Jahr eine Initiative, die seltene Allergien unter den Nationalen Aktionsplan für seltene Erkrankungen stellen soll. Wir werden Sie hierüber weiter auf dem Laufenden halten.

Nicht zuletzt möchten wir Sie zum 12. Deutschen Allergiekongress (DAK) vom 5. bis 7. Oktober 2017 in Wiesbaden einladen: Mehr Allergologie als Sie auf dem DAK geboten bekommen, geht definitiv nicht! Die Abstracts zum Allergiekongress finden Sie ab Seite 55 in dieser Allergo-Journal-Ausgabe. Diese Fortbildungsveranstaltung findet auf höchstem Niveau statt und bietet eine Übersicht über die aktuellen, wissenschaftlichen Entwicklungen in Verbindung mit praktischer Fortbildung und zahlreichen Workshops. Treffen Sie in Wiesbaden alte und neue Freunde und lassen Sie sich von der Allergologie neu begeistern!

Viel Freude beim Lesen dieses Heftes und herzlich willkommen auf dem 12. Deutschen Allergiekongress in Wiesbaden!

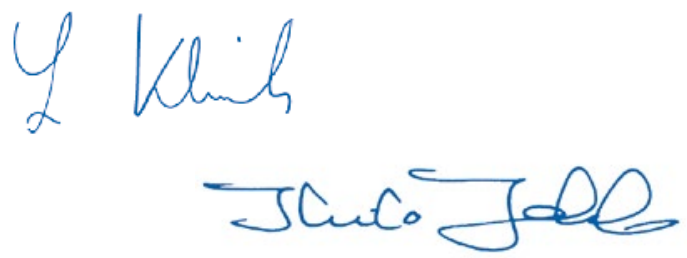

